\title{
Origins Space Telescope: baseline mission concept
}

\begin{abstract}
David Leisawitz $\odot$, ${ }^{\mathrm{a}, *}$ Edward Amatucci $\odot,{ }^{\mathrm{a}}$ Lynn Allen, ${ }^{\mathrm{b}}$ Jonathan Arenberg $\odot$, ${ }^{\mathbf{c}}$ Lee Armus, ${ }^{\text {d }}$ Cara Battersby $\odot,{ }^{\mathbf{e}}$ James Bauer, ${ }^{\mathbf{f}}$ Bobby G. Beaman, ${ }^{\text {a,g }}$ Ray Bell, ${ }^{\text {h }}$ Porfirio Beltran, ${ }^{\text {a }}$ Dominic Benford $\odot,{ }^{\text {i }}$ Edward Bergin, jeffrey Bolognese, ${ }^{\text {j }}$ Charles M. Bradford, ${ }^{k}$ Damon Bradley, ${ }^{\text {a }}$ Denis Burgarella $\odot$, Sean Carey, ${ }^{\text {d }}$ Ruth Carter, ${ }^{\mathrm{a}}$
\end{abstract} J. D. (Danny) Chi, ${ }^{\text {c }}$ Asantha Cooray, ${ }^{\mathrm{m}}$ James Corsetti, ${ }^{\text {a }}$ Thomas D'Asto, ${ }^{\text {a,n }}$ Elvire De Beck $\odot,{ }^{\circ}$ Kevin Denis, ${ }^{a}$ Christopher Derkacz, ${ }^{\text {a,p }}$ Larry Dewell, ${ }^{\text {h }}$ Michael DiPirro, ${ }^{\text {a }}$ Cleland P. Earle, ${ }^{\text {a,f }}$ Matthew East, ${ }^{\mathrm{b}}$ Samantha Edgington, Kimberly Ennico $\odot,{ }^{\mathrm{q}}$ Louis Fantano, ${ }^{\mathrm{a}}$ Greg Feller, ${ }^{\mathrm{r}}$ David Folta, ${ }^{\mathrm{a}}$ Jonathan Fortney, ${ }^{\mathrm{s}}$ Benjamin J. Gavares, ${ }^{\text {a,t }}$ Joseph Generie, ${ }^{\mathrm{a}}$ Maryvonne Gerin $\odot,{ }^{\text {u Zachary Granger, }}$, Thomas P. Greene $\odot,{ }^{\mathrm{q}}$ Alex Griffiths $\odot,{ }^{\text {v }}$ George Harpole, ${ }^{\text {c }}$ Keith Harvey, ${ }^{\text {b }}$ Frank Helmich $\odot,{ }^{\text {w }}$ Lawrence Hilliard, ${ }^{a}$ Joseph Howard $\odot,{ }^{\text {a }}$ Michael Jacoby, Anisa Jamil, ${ }^{\text {a }}$ Tracee Jamison, ${ }^{a}$ Lisa Kaltenegger, ${ }^{\mathrm{x}}$ Tiffany Kataria, ${ }^{\mathrm{k}}$ John S. Knight, ${ }^{\mathrm{y}}$ Perry Knollenberg, ${ }^{\text {c }}$ Charles Lawrence, ${ }^{\mathrm{k}}$ Paul Lightsey $\odot{ }^{\mathrm{y}}$ Sarah Lipscy, Eric Mamajek $\odot,{ }^{k}$ Gregory Martins, ${ }^{\text {a,n }}$ John C. Mather $\odot,{ }^{a}$ Margaret Meixner, ${ }^{\mathrm{z}}$ Gary Melnick, ${ }^{\text {aa }}$ Stefanie Milam $\odot,{ }^{\mathrm{a}}$ Ted Mooney, Samuel H. Moseley, ${ }^{\text {ab }}$ Desika Narayanan, ${ }^{\text {ac }}$ Susan Neff, ${ }^{\text {a }}$ Thanh Nguyen, ${ }^{c}$ Alison Nordt, ${ }^{\text {h }}$ Jeffrey Olson $\odot$, ${ }^{\text {h Deborah Padgett, }}{ }^{\mathrm{k}}$ Michael Petach, Susanna Petro, ${ }^{\text {a John Pohner, }}{ }^{\text {c }}$ Klaus Pontoppidan $\odot,{ }^{\text {ad }}$ Alexandra Pope $\odot,{ }^{\text {ae }}$ Daniel Ramspacker, ${ }^{a}$ Alison Rao, ${ }^{\text {a }}$ Thomas Roellig, ${ }^{\mathrm{q}}$ Itsuki Sakon $\odot,{ }^{\text {af }}$ Carly Sandin, ${ }^{\text {a }}$ Karin Sandstrom $\odot,{ }^{\text {ag }}$ Douglas Scott $\odot,{ }^{\text {ah }}$ Len Seals $\odot,{ }^{\text {a }}$

Kartik Sheth ${ }^{\text {, }}{ }^{\text {L Lawrence M. Sokolsky, }}{ }^{\text {h }}$ Johannes Staguhn $\odot$, a,ai

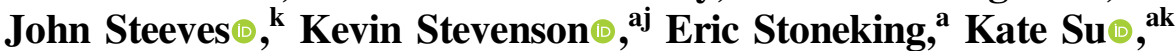
Kiarash Tajdaran, ${ }^{\mathrm{h}}$ Steven Tompkins, ${ }^{\text {a Joaquin Vieira, }},{ }^{\text {al }}$

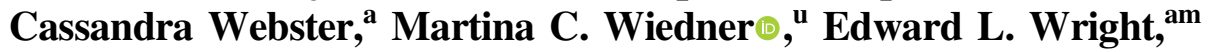
Chi $\mathrm{Wu}^{\text {a }}$ and Jonas Zmuidzinas ${ }^{\text {an }}$

${ }^{a}$ NASA Goddard Space Flight Center, Greenbelt, Maryland, United States

${ }^{b}$ L3Harris Technologies, Rochester, New York, United States

${ }^{\mathrm{c}}$ Northrop-Grumman Space Systems, Redondo Beach, California, United States

${ }^{\mathrm{d} C}$ California Institute of Technology, Infrared Processing and Analysis Center, Pasadena, California, United States

${ }^{\mathrm{e}}$ University of Connecticut, Department of Physics, Storrs, Connecticut, United States fUniversity of Maryland, Astronomy Department, College Park, Maryland, United States ${ }^{g}$ Science Systems and Applications, Inc., Lanham, Maryland, United States

${ }^{h}$ Lockheed-Martin Advanced Technology Center, Palo Alto, California, United States ${ }^{i}$ Astrophysics Division, Science Mission Directorate, NASA Headquarters, Washington, DC, United States

${ }^{\mathrm{j}}$ University of Michigan, Department of Astronomy, Ann Arbor, Michigan, United States

${ }^{\mathrm{k}}$ Jet Propulsion Laboratory, California Institute of Technology, Pasadena, California, United States

${ }^{1}$ Laboratoire d'Astrophysique de Marseille, Marseille, France

${ }^{\mathrm{m}}$ University of California, Department of Physics and Astronomy, Irvine, California, United States

${ }^{n}$ ATA Aerospace Civil, Greenbelt, Maryland, United States

${ }^{\circ}$ Chalmers Institute of Technology, Department of Space, Earth and Environment, Gothenburg, Sweden

pInuTeq, LLC, Beltsville, Maryland, United States

*Address all correspondence to D. Leisawitz, david.t.leisawitz@nasa.gov 
${ }^{\mathrm{q}}$ NASA Ames Research Center, Mountain View, California, United States

${ }^{\mathrm{r}}$ Coherent, Inc., Santa Clara, California, United States

${ }^{s}$ University of California, Astronomy and Astrophysics Department, Santa Cruz, California, United States

'Orbital ATK, Dulles, Virginia, United States

"Sorbonne Université, Observatoire de Paris, CNRS, LERMA, Paris, France

${ }^{v}$ University of Nottingham, School of Physics and Astronomy, Nottingham, United Kingdom

${ }^{\text {w}}$ SRON Netherlands Institute for Space Research and Groningen University, Groningen,

The Netherlands

${ }^{\mathrm{x}}$ Cornell University, The Department of Astronomy, Ithaca, New York, United States

${ }^{\mathrm{y}}$ Ball Aerospace Corporation, Boulder, Colorado, United States

${ }^{\mathrm{z}}$ Universities Space Research Association, Columbia, Maryland, United States

${ }^{a a}$ Harvard-Smithsonian Center for Astrophysics, Cambridge, Massachusetts, United States

${ }^{a b}$ Quantum Circuits, Inc., New Haven, Connecticut, United States

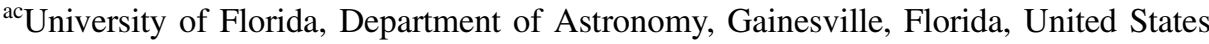

${ }^{a d}$ Space Telescope Science Institute, Baltimore, Maryland, United States

${ }^{a}$ University of Massachusetts, Department of Astronomy, Amherst, Massachusetts, United States

${ }^{\text {af }}$ The University of Tokyo, School of Science, Tokyo, Japan

${ }^{\mathrm{ag}}$ University of California, CASS, San Diego, California, United States

${ }^{a h}$ University of British Columbia, Department of Physics and Astronomy, Vancouver, British Columbia, Canada

ai Johns Hopkins University, Department of Physics and Astronomy, Baltimore, Maryland, United States

ajJohns Hopkins University, Applied Physics Laboratory, Laurel, Maryland, United States

${ }^{\mathrm{ak}}$ The University of Arizona, Department of Astronomy and Steward Observatory, Tucson, Arizona, United States

${ }^{a l}$ University of Illinois, Department of Astronomy, Urbana-Champaign, Urbana, Illinois, United States

${ }^{\mathrm{am}}$ University of California Los Angeles, Division of Astronomy and Astrophysics, Los Angeles, California, United States

${ }^{\text {an }}$ California Institute of Technology, Division of Physics, Mathematics and Astronomy, Pasadena, California, United States

Abstract. The Origins Space Telescope will trace the history of our origins from the time dust and heavy elements permanently altered the cosmic landscape to present-day life. How did galaxies evolve from the earliest galactic systems to those found in the Universe today? How do habitable planets form? How common are life-bearing worlds? To answer these alluring questions, Origins will operate at mid- and far-infrared (IR) wavelengths and offer powerful spectroscopic instruments and sensitivity three orders of magnitude better than that of the Herschel Space Observatory, the largest telescope flown in space to date. We describe the baseline concept for Origins recommended to the 2020 US Decadal Survey in Astronomy and Astrophysics. The baseline design includes a 5.9-m diameter telescope cryocooled to $4.5 \mathrm{~K}$ and equipped with three scientific instruments. A mid-infrared instrument (Mid-Infrared Spectrometer and Camera Transit spectrometer) will measure the spectra of transiting exoplanets in the 2.8 to $20 \mu \mathrm{m}$ wavelength range and offer unprecedented spectrophotometric precision, enabling definitive exoplanet biosignature detections. The far-IR imager polarimeter will be able to survey thousands of square degrees with broadband imaging at 50 and $250 \mu \mathrm{m}$. The Origins Survey Spectrometer will cover wavelengths from 25 to $588 \mu \mathrm{m}$, making wide-area and deep spectroscopic surveys with spectral resolving power $R \sim 300$, and pointed observations at $R \sim 40,000$ and 300,000 with selectable instrument modes. Origins was designed to minimize complexity. The architecture is similar to that of the Spitzer Space Telescope and requires very few deployments after launch, while the cryothermal system design leverages James Webb Space Telescope technology and experience. A combination of current-state-of-the-art cryocoolers and next-generation detector technology will enable Origins' natural background-limited sensitivity. () The Authors. Published by SPIE under a Creative Commons Attribution 4.0 Unported License. Distribution or reproduction of this work in whole or in part requires full attribution of the original publication, including its DOI. [DOI: 10.1117/1.JATIS.7.1.011002] 
Keywords: infrared; space telescope; cryogenic; spectroscopy; galaxy evolution; planet formation; biosignatures.

Paper 20072SS received Jun. 13, 2020; accepted for publication Dec. 1, 2020; published online Jan. 6, 2021.

\section{Introduction}

In astrophysics, the far-infrared (IR), wavelengths from about 30 to $600 \mu \mathrm{m}$, is information-rich, and to this day, vastly underexploited. With past far-IR space missions, the Herschel Space Observatory $^{1}$ and its predecessors, notably the Infrared Astronomical Satellite, ${ }^{2}$ the Cosmic Background Explorer (COBE) ${ }^{3}$ the Infrared Space Observatory, ${ }^{4}$ the Spitzer Space Telescope,${ }^{5}$ and AKARI, ${ }^{6}$ the astrophysics community made tremendous scientific strides and surprising discoveries. We gained insight into the role magnetic fields play in the star-formation process and the composition and energetics of the interstellar medium, and we characterized the cosmic infrared background. Stunningly, among many achievements, Spitzer gave us empirical evidence of weather on an exoplanet. Nevertheless, with their limited angular resolution, past far-IR telescopes quickly reached the confusion noise limit at which source crowding prevents individual objects from being discernable in an image. Even Herschel, the largest telescope flown in space to date, offered diffraction-limited angular resolution comparable only to that of the telescopes used by Galileo at visible wavelengths four centuries ago.

Improved measurement capabilities in the far-IR will enable a great deal more to be accomplished. We already know that interstellar dust formed early, and thereafter permanently altered the process of star formation. The dust absorbs and blocks UV/visible starlight and emits in the far-IR. The far-IR is replete with spectral lines from water vapor, the dominant coolant of the interstellar medium, and important diagnostics of the hardness of the interstellar radiation field, and broad-band spectral features of water ice. With a future far-IR telescope, we will learn how the conditions for habitability sometimes arise during the planet-formation process. How does water make its way from the interstellar medium to a planet warm enough to melt ice, but not so hot as to result in its complete evaporation? Additionally, we will characterize the physical and chemical conditions in nascent galaxies, complementing the Webb Telescope's observations of ancient starlight, ${ }^{7}$ to understand how galaxies changed throughout cosmic history. To access the information available in these diagnostics, the astrophysics community needs a far-IR space telescope that approaches natural background sensitivity limits with moderate to high-resolution spectroscopy.

To prepare for the US National Academies' 2020 Decadal Survey in Astronomy and Astrophysics, NASA sponsored a study of the Origins Space Telescope (hereafter Origins). The study was conducted from December 2015 to August 2019, when a final report was delivered to the Decadal Survey committee. The Origins Science and Technology Definition Team (STDT) prioritized scientific objectives attainable with a telescope that provides superlative sensitivity but does not require a large improvement in angular resolution relative to the $3.5-\mathrm{m}$ Herschel telescope. Thus, the STDT decided early on that Origins would be a single-aperture telescope. A companion paper to this one describes design trades and the rationale for the STDT's choices. ${ }^{8}$

This paper gives an overview of the Origins baseline mission concept, while parallel papers describe many different facets of the mission study: the scientific motivation for the mission and derived requirements; ${ }^{9}$ the cryothermal system design and the attainability of the $4.5 \mathrm{~K}$ optical system operating temperature with current state-of-the-art cryocooler technology; ${ }^{10}$ the telescope optical design and wavefront error budget: ${ }^{11}$ materials trades, choices, and potential alternatives; ${ }^{12}$ four scientific instruments ${ }^{13-16}$ (one of which is optional and not included in the baseline mission concept); the integration and test program $;{ }^{17}$ and key enabling technologies, notably cryocoolers, ${ }^{18}$ mid-infrared detectors, ${ }^{19}$ far-IR detectors, ${ }^{19-23}$ and the far-IR detector readout system. ${ }^{24}$ Earlier papers presented preliminary results of a stray-light analysis of the telescope ${ }^{25}$ and a pupil densification technique that can be used to mitigate the effects of pointing jitter and enable extremely precise spectroscopic measurements of transiting exoplanets to search for planets with biosignatures in the mid-IR ( 2.8 to $25 \mu \mathrm{m}){ }^{26}$ 
This paper is organized as follows. Section 2 contains a synopsis of the scientific motivation for the mission and the flowdown from objectives to top-level derived requirements. Section 3 presents the baseline mission concept. The schedule and estimated cost are given in Secs. 3.7 and 3.8, respectively, and the mission's estimated performance is described in Sec. 4. We summarize in Sec. 5.

\section{Science Goals, Objectives, and Flowdown to Measurement Requirements}

In consultation with the scientific community, the Origins STDT prioritized three goals for the mission. These established priorities are motivated by their profound significance and likely durability in light of expected advances from, and limitations of current and nextgeneration observatories [the Atacama Large Millimeter Array (ALMA) ${ }^{27}$ the Vera C. Rubin Observatory, ${ }^{28}$ JWST, and the Nancy Grace Roman Space Telescope $\left.{ }^{29}\right]$. Origins science featured prominently in the science white papers the community submitted to the Astro2020 Decadal Survey. The Origins mission goals also align directly with the three themes of NASA's Astrophysics program: How does the Universe work? How did we get here? and Are we alone ${ }^{30}$ Corresponding to each mission goal are three distinct scientific objectives. Figure 1 summarizes the goals and objectives of Origins and their relationship to NASA themes.

The nine objectives listed in the last row of Fig. 1 collectively have driven the Origins measurement requirements and, in turn, the baseline mission design, as shown at a high level in Fig. 2. The measurements can only be made with a telescope in space because the Earth's atmosphere absorbs most of the light at the wavelengths of interest (Fig. 3). The measurements require superlative sensitivity, and thus, a large cold telescope. Figure 4 shows the need for a telescope temperature below $6 \mathrm{~K}$ to suppress the observatory's thermal self-emission and reach a noise level close to the natural astrophysical background (consisting of zodiacal emission from the solar system, dust emission from the Milky Way, and the cosmic microwave background at the longest wavelengths). ${ }^{31}$ The telescope must also be large enough for the community to achieve its objectives in a reasonable mission lifetime, driving the need for a primary mirror at least $5.3 \mathrm{~m}$ in diameter, below which the search for biosignatures in exoplanet atmospheres would be significantly compromised (Fig. 5). The prioritized scientific objectives do not require high (subarcsec) angular resolution, and our studies found that the telescope could be diffraction limited at $30 \mu \mathrm{m}$.

As described in Sec. 3, three science instruments satisfy the Origins spectroscopic and imaging requirements. For observations of galaxies and protoplanetary disks, the far-IR $(\lambda>25 \mu \mathrm{m})$ instruments have to deliver spectroscopic data with resolving power $(R=\lambda / \Delta \lambda)$ ranging from 3 to 43,000 in approximately order-of-magnitude increments, and $R>200,000$ in

\begin{tabular}{|l|l|l|l|}
\hline NASA Goal & How Does the Universe Work? & $\begin{array}{l}\text { How do galaxies form stars, } \\
\text { make metals, and grow their } \\
\text { central supermassive black holes } \\
\text { from reionization to today? }\end{array}$ & $\begin{array}{l}\text { How do the conditions for } \\
\text { Science } \\
\text { Goals }\end{array}$
\end{tabular}

Fig. 1 Mission design scientific drivers for the Origins Space Telescope. 


\begin{tabular}{|c|c|c|c|c|}
\hline \multicolumn{2}{|c|}{ Origins Science Driver } & \multicolumn{3}{|c|}{ Technical or instrument parameter } \\
\hline Scientific goal & Observable & Parameter & Requirement & Design \\
\hline \multirow{2}{*}{$\begin{array}{l}\text { How do galaxies } \\
\text { form stars, make } \\
\text { metals, and grow } \\
\text { their central } \\
\text { SMBHs? }\end{array}$} & \multirow{2}{*}{$\begin{array}{l}\text { Mid-and far- } \mathbb{R} \\
\text { rest-frame spectral } \\
\text { lines. }\end{array}$} & Aperture size & $>3.0-5.0 \mathrm{~m}$ & $5.9 \mathrm{~m}$ \\
\hline & & $\begin{array}{l}\text { Telescope } \\
\text { temperature }\end{array}$ & $<6 K$ & $4.5 \mathrm{~K}$ \\
\hline \multirow{4}{*}{$\begin{array}{l}\text { How do the } \\
\text { conditions for } \\
\text { habitability } \\
\text { develop during the } \\
\text { process of planet } \\
\text { formation? }\end{array}$} & $\begin{array}{l}\mathrm{H}_{2}^{18} 0110-101 \\
547.4-\mu \mathrm{m} \text { line }\end{array}$ & $\lambda_{\max }$ & $>550 \mu \mathrm{m}$ & $588 \mu \mathrm{m}$ \\
\hline & $\begin{array}{c}\mathrm{H}_{2} \mathrm{O} 212-101 \\
179.5-\mu \mathrm{m} \text { line }\end{array}$ & $R=\lambda / \Delta \lambda$ & $>200,000$ & 202,785 \\
\hline & \multirow[t]{2}{*}{ HD 1-0 112- $\mu \mathrm{m}$ line } & $\begin{array}{l}\text { Spectral line } \\
\text { sensitivity }\end{array}$ & $\begin{array}{l}10^{-20} \mathrm{~W} \mathrm{~m}^{-2} \\
(1 \mathrm{hr} ; 5 \sigma)\end{array}$ & $\begin{array}{c}5 \times 10^{-27} \mathrm{~W} \mathrm{~m}^{-2} \\
(1 \mathrm{hr} ; 50)\end{array}$ \\
\hline & & $R=\lambda / \Delta \lambda$ & $>40,000$ & 43,000 \\
\hline \multirow{2}{*}{$\begin{array}{l}\text { Do planets orbiting } \\
\text { M-dwarf stars } \\
\text { support life? }\end{array}$} & \multirow{2}{*}{$\begin{array}{c}\mathrm{CH}_{4}(3.3 \& 7.4 \mu \mathrm{m}), \\
\mathrm{N}_{2} \mathrm{O}(4.5 \& 7.8 \mu \mathrm{m}), \\
\mathrm{O}_{3}(9.7 \mu \mathrm{m}), \mathrm{CO}_{2} \\
(4.3 \& 15 \mu \mathrm{m}), \mathrm{H}_{2} \mathrm{O} \\
(6.3,17+\mu \mathrm{m})\end{array}$} & $\lambda_{\min }$ & $<3 \mu \mathrm{m}$ & $2.8 \mu \mathrm{m}$ \\
\hline & & Aperture size & $>5.3 \mathrm{~m}$ & $5.9 m$ \\
\hline
\end{tabular}

Fig. 2 Summary of Origins requirements.

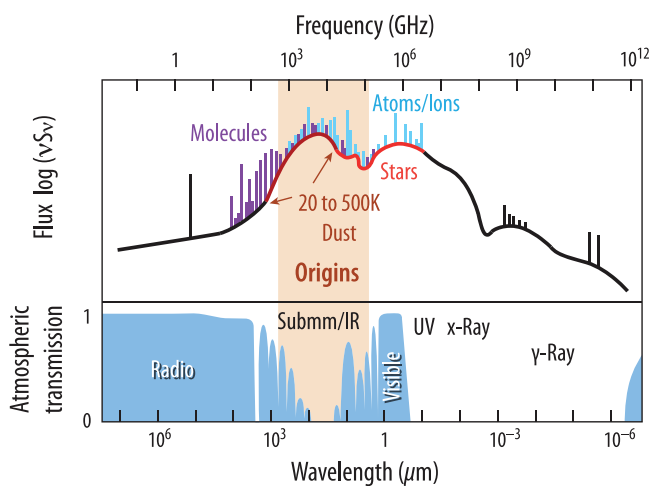

Fig. 3 The spectrum of a typical galaxy (top) bears signatures of dust thermal emission and spectral lines from molecules, atoms, and ions, which probe key astrophysical processes, such as star formation and physical conditions in the interstellar medium. Spectra like this one are Dopplershifted toward longer wavelengths (leftward in the graph) by the expansion of the Universe appropriate for the galaxy's distance. Origins was designed to operate in the wavelength range 2.8 to $588 \mu \mathrm{m}$, which is only partly accessible from the ground in some atmospheric windows (bottom).

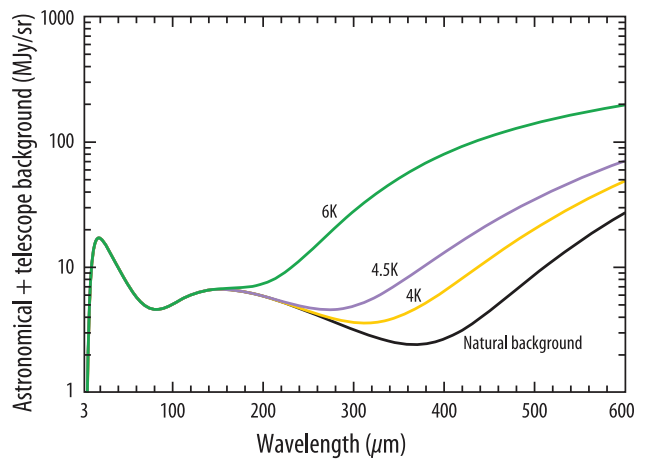

Fig. 4 Background emission from a cold telescope is strongly dependent on the telescope temperature and only approaches the natural background (zodiacal and Galactic dust emission and the cosmic microwave background) and satisfies Origins science requirements when the optical system components are colder than $6 \mathrm{~K}$. Here, we assume a telescope emissivity of $2 \%$. As noted in Sec. 3, the Origins telescope is cooled to $4.5 \mathrm{~K}$, since current state-of-the-art cryocoolers have a cold stage at this temperature. 


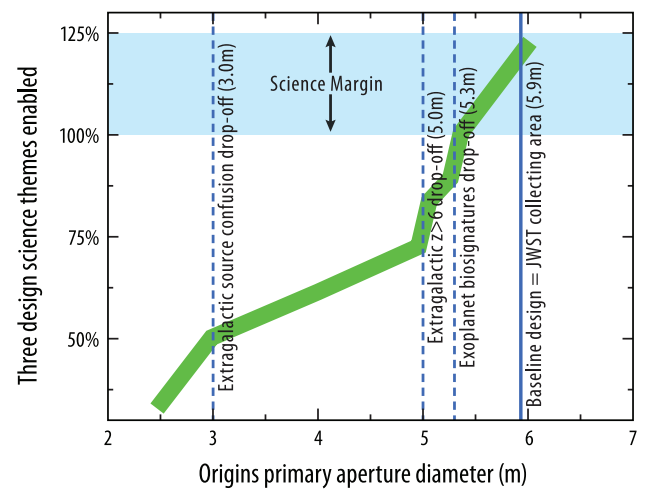

Fig. 5 To achieve the highest priority scientific objectives, the Origins telescope must be cold (Fig. 4) but also have a primary aperture at least $5.3 \mathrm{~m}$ in diameter. This requirement comes primarily from the exoplanet science case to detect biosignatures in a five-year mission (given that planet transit durations are fixed and the sensitivity cannot be recovered with a longer singleepoch integration, unlike most other proposed Origins observations). The prioritized extragalactic study places an aperture size requirement of $>5 \mathrm{~m}$, based on the need to detect a statistically significant sample of galaxies at redshift $z>6$, to study the formation mechanisms and physical properties of dust and heavy elements during the epoch of reionization. The minimum primary aperture diameter is $3 \mathrm{~m}$ to enable an effective extragalactic and Galactic science program, so that source confusion does not compromise the telescope's ability to conduct spectroscopic studies of galaxies at $z=2$ to 3 . A 3-m or larger aperture is needed also to study water and the total gas content of proto-planetary disks at the distance of Orion. To estimate the percentage of science enabled, the Origins STDT evaluated an observing program developed in collaboration with astronomical community members, resulting in representative measurements that would be made to achieve the prioritized Origins scientific objectives. Depending on the telescope size, individual observations can be carried out fully as proposed or only in part, or in some cases may not be possible at all or can be executed better than planned. The ordinate in this graph shows the overall fraction of science enabled.

the spectral range 100 to $200 \mu \mathrm{m}$. A mid-IR instrument has to provide superlative stability ( $<5 \mathrm{ppm}$ ) and $R \sim 50$ to 300 in the spectral range 2.8 to $20 \mu \mathrm{m}$ to enable a fruitful search for biosignatures (e.g., the simultaneous presence in an atmosphere of $\mathrm{O}_{3}$ with either $\mathrm{CH}_{4}$ or $\mathrm{N}_{2} \mathrm{O}$ ) in the spectra of transiting exoplanets. To achieve its extragalactic objectives, the telescope also has to be able to survey thousands of square degrees of sky. The science case for Origins is described in detail and a comprehensive Science Traceability Matrix is presented in a companion paper. ${ }^{9}$

To summarize, Origins was designed to trace our cosmic history, from the formation of the first galaxies and the rise of heavy elements to the development of habitable worlds and presentday life. It achieves its scientific objectives through exquisite sensitivity to infrared radiation from ions, atoms, molecules, dust, water vapor, and ice, and observations of extra-solar planetary atmospheres, protoplanetary disks, and large-area extragalactic fields in the wavelength range 2.8 to $588 \mu \mathrm{m}$ with a large $(>5.3 \mathrm{~m})$ cold $(<6 \mathrm{~K})$ telescope and three instruments.

\section{Mission Design}

Origins is a NASA-led mission, managed by a NASA Center, and includes domestic and international partners. We applied NASA guidelines for the 2020 Decadal Survey in Astronomy and Astrophysics and grounded in NASA and industry experience from previous successful large Class A missions to develop the Origins baseline mission design.

\subsection{Key decisions}

Noting that three large launch vehicles are presently under study or development, NASA's Space Launch System (SLS) and commercially developed alternatives, the Origins study team decided 
that the benefits of greatly reduced complexity (a telescope that does not have to be deployed in space) outweighed the unlikely possibility that none of these launch vehicles would exist by the mid-2030s, when Origins would fly. The decision to design for compatibility with an 8.4-m diameter fairing on the SLS enabled the study team to adopt the proven Spitzer cryothermal system architecture and an on-axis telescope with a 5.9-m diameter primary mirror, exceeding the 5.3-m minimum size required, while allowing room in the fairing for a simply deployable two-layer sunshade. An obstructed circular primary mirror this size would have the same lightcollecting area as JWST $\left(25 \mathrm{~m}^{2}\right)$ and provide margin over the minimum size required to achieve currently prioritized Origins science objectives (Fig. 5), and robustness to the possibility that science priorities will evolve in response to progress made with other observing facilities. As noted below, on-orbit servicing could extend the mission lifetime and contribute to the robustness of the mission to unforeseen scientific developments.

Advances in cryocooler technology for JWST and Hitomi also enabled this design solution. Expendable cryogens for telescope cooling would not only limit the mission's lifetime, but would increase the mass and volume of the observatory. Cryocoolers have demonstrated reliability in space and are much less massive and voluminous than a Dewar full of expendable cryogen. Existing cryocoolers have a cold stage at $4.5 \mathrm{~K},{ }^{10}$ so this temperature was adopted for the telescope, exceeding the design requirement and adding sensitivity margin at the longest Origins wavelengths (Fig. 4). Four current-state-of-the-art cryocoolers will cool the telescope, with $100 \%$ margin in heat lift capacity at each temperature stage. Table 1 shows the major observatory-level design parameters.

\subsection{Simple Deployments After Launch}

With its Spitzer-like architecture (Fig. 6), Origins requires only a few simple deployments to transform from stowed to operational configuration. A cold cylindrical shield (35-K barrel and $4.5-\mathrm{K}$ baffle) surrounds the telescope, and the shield is protected from sunlight by a two-layer sunshade. The sunshade provides passive cooling. As noted above, the optical system launches in its operational configuration, requiring no mirror, cold shield, or baffle deployments after launch. Only the communication antenna, solar array, telescope cover, and sunshades are deployed. These deployments rely on mechanisms with extensive heritage and are considered low risk. The sunshade layers deploy like a pop-up tent with spring-loaded poles; stored energy in flexible rods pulls the shade material into its desired shape. Telescoping arms place each of the two sunshades at their intended distances from the cold shield. The sunshade deployment sequence can be tested on the ground in existing facilities.

Table 1 Origins observatory-level parameters.

\begin{tabular}{ll}
\hline \hline Mission parameter & \multicolumn{1}{c}{ Value } \\
\hline Telescope: aperture diameter/area & $5.9 \mathrm{~m} / 25 \mathrm{~m}^{2}$ \\
Telescope: diffraction limited at & $30 \mu \mathrm{m}$ \\
Telescope: temperature & $4.5 \mathrm{~K}$ \\
Wavelength coverage & 2.8 to $588 \mu \mathrm{m}$ \\
Maximum scanning speed & $60^{\prime \prime}$ per second \\
Mass: dry/wet (with margin) & $12,000 \mathrm{~kg} / 13,000 \mathrm{~kg}$ \\
Power (with margin) & $4800 \mathrm{~W}$ \\
Launch year & 2035 \\
Launch vehicle (large vehicle) & SLS or equivalent commercial vehicle \\
Orbit & Sun-Earth L2 \\
Propellant lifetime & 10 years, serviceable \\
\hline \hline
\end{tabular}



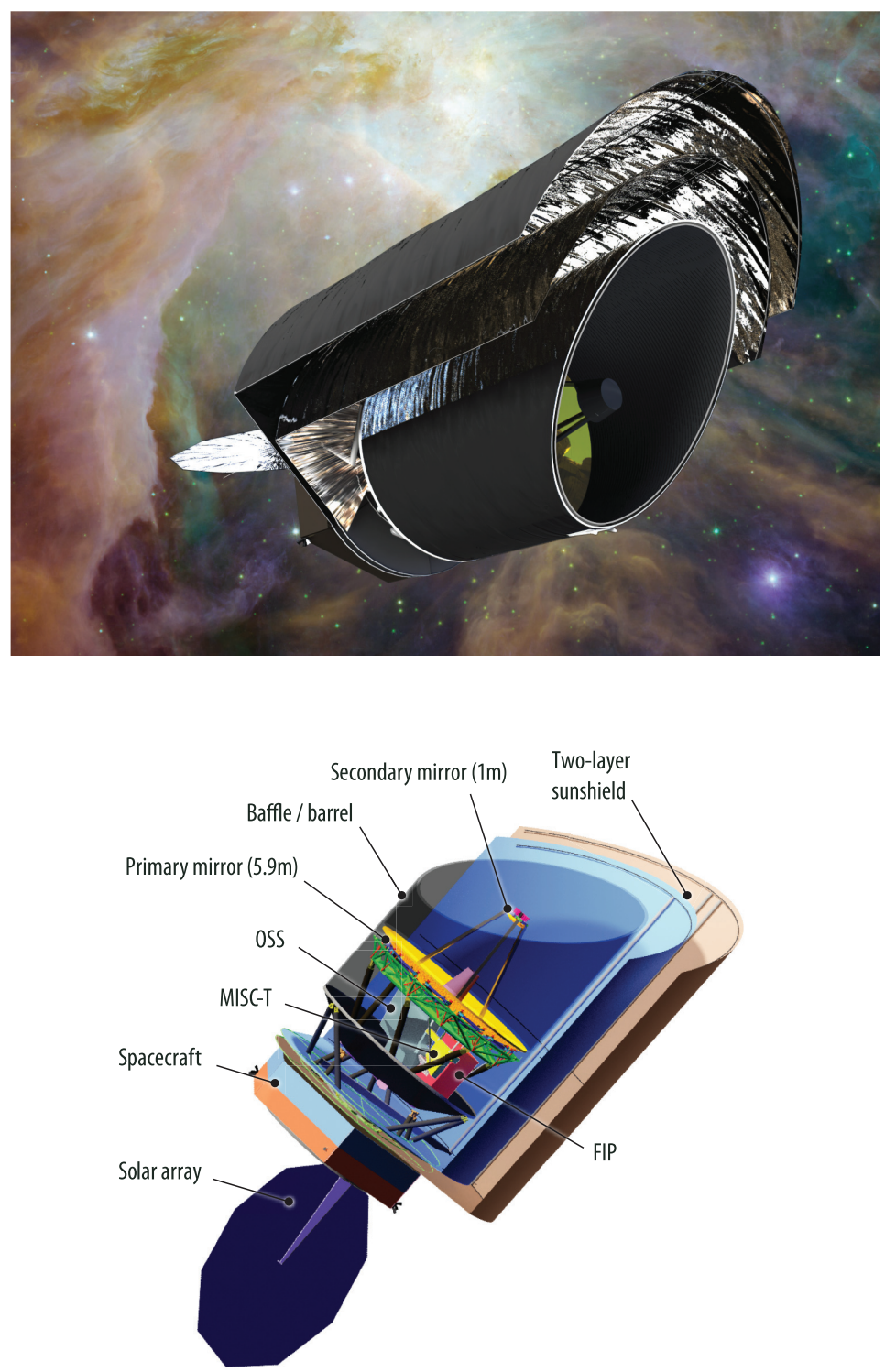

Fig. 6 Origins (artist's concept, top) builds on substantial heritage from Spitzer to minimize assembly, integration and testing, and deployment risks. A cutaway view (bottom) shows major elements of the flight system, including the instruments: the Origins Survey Spectrometer (OSS) is a far-IR spectrometer; ${ }^{13}$ FIP is a far-IR imager and polarimeter; ${ }^{14}$ and MISC-T is a mid-infrared transiting exoplanet spectrometer. ${ }^{15}$ Detailed descriptions of the optical and cryo-thermal systems can be found in companion papers by Corsetti et al. ${ }^{11}$ and DiPirro et al., ${ }^{10}$ respectively, where the instrument configuration will be clearer.

\subsection{Telescope}

The telescope is a three-mirror anastigmat (TMA) with an on-axis secondary. ${ }^{11}$ The TMA design form is well understood and low risk, having been proven in testing for JWST. The telescope is diffraction-limited at $30 \mu \mathrm{m}$ and used as a light bucket at shorter wavelengths, where a compact point spread function (PSF) is not required. The PSF at $\lambda<30 \mu \mathrm{m}$ is essentially the same as the PSF at $30 \mu \mathrm{m}$, and it varies slightly at all wavelengths across the telescope field of view (FOV).

An inner ring of six keystone-shaped segments, and an outer ring of twelve similarly shaped segments, comprise the primary mirror. The segments in the outer annulus are identical, while those in the inner annulus are interchangeable until notches are cut in opposite outer corners to 
accommodate the secondary mirror struts. This segmentation approach reduces design, manufacturing, validation, and verification time, and keeps the number of required flight spare units to a minimum. The JWST primary mirror segment actuator design is adopted to allow the Origins primary mirror segments to be adjusted in three degrees of freedom (tip, tilt, and piston). Far-IR Imager Polarimeter (FIP) images of a point source, such as a quasar, will be used to provide in-flight feedback on mirror-segment alignment. A detailed alignment plan will be developed during Phase A. We expect to make these adjustments only once on orbit, during the commissioning period. The telescope's mirrors and mirror segments can be diamond-turned and rough-polished to the required precision in existing facilities. The mirrors do not require time-consuming cryo-null figuring because the mirrors will retain shapes within the range of their specifications when they are cooled.

A flat field-steering mirror (FSM) follows the telescope's three powered mirrors. The FSM controls the optical line of sight to map small fields and/or modulate the signal on the detectors, and it suppresses internal disturbances below $10 \mathrm{~Hz}$. Its size, mass, and range of motion are similar to those of the JWST Fine Steering Mirror. The Origins FSM could use the same actuators as JWST, with the addition of superconducting coils to limit heat dissipation.

\subsection{Instruments}

Three science instruments spanning the wavelength range 2.8 to $588 \mu \mathrm{m}$ give Origins the spectroscopic and imaging capabilities required to achieve the mission's scientific objectives (Fig. 1). The operating modes and measurement capabilities of these instruments are summarized in Table 2.

The Origins Survey Spectrometer (OSS) uses six gratings to take multi-beam spectra simultaneously across the 25 to $588 \mu \mathrm{m}$ window through long slits, enabling deep three-dimensional (3D) extra-galactic surveys. ${ }^{13}$ The six slits overlap on the sky so that a point source couples to all six bands simultaneously. When needed, a Fourier transform spectrometer and an etalon provide high and ultrahigh spectral resolving power, respectively. These high-resolution modes are essential for studies of water and the gas-mass-tracing hydrogen deuteride (HD) emission lines in protoplanetary disks.

The confusion limit has been a fundamental barrier for deep imaging surveys with past far-IR missions. Confusion is much less of a problem for OSS, as the spectra for each galaxy in the field will be used to deblend galaxies within a given beam. Confusion occurs when spectral lines from foreground sources overlap in the beam of a high-redshift target. We used galaxy counts, ${ }^{32}$ coupled with constraints on the line-to-IR luminosity ratios ${ }^{33}$ to calculate line confusion at the depths of the STDT's proposed OSS extragalactic surveys. Figure 7 shows the integral line counts per spatial beam and spectral resolution element compared to the depths of the deep survey in each of the six OSS bands. Spectral confusion is an issue when the number of sources per beam is $>1 / 15$ (horizontal dotted line in Fig. 7). At the depths of the planned deep survey (dashed vertical lines), spectral line confusion is not expected to limit the ability of these surveys to achieve Origins' primary science objectives.

The Far-IR Imager Polarimeter (FIP) provides imaging and polarimetric measurement capabilities at 50 and $250 \mu \mathrm{m} .{ }^{14}$ Its fast mapping enables rapid follow-up of transient or variable sources and efficient monitoring campaigns. FIP surveys take advantage of Origins' agility. Like Herschel, Origins can scan-map the sky at 60" per second. Fast scanning is essential, since the FIP $250-\mu \mathrm{m}$ channel reaches the extragalactic source confusion limit in a few milliseconds. FIP will enable wide area ( $\left.\geq 1000 \mathrm{deg}^{2}\right)$ photometric surveys, leading to large statistical multiwavelength studies of populations of astronomical objects, complementing the Vera C. Rubin Observatory and the Nancy Grace Roman Space Telescope. Origins will enable the astronomical community to thoroughly explore the currently unknown faint, far-IR Universe.

The Mid-Infrared Spectrometer and Camera Transit spectrometer (MISC-T) measures $R=50$ to 300 spectra in the 2.8 to $20 \mu \mathrm{m}$ range with three simultaneously operating bands. ${ }^{15}$ MISC-T provides exquisite stability and precision (5 ppm between 2.8 and $10 \mu \mathrm{m}$ ) for exoplanet transits. It employs pupil densification to mitigate the effects of observatory jitter and relies on a detector stability improvement relative to current state-of-the-art levels. ${ }^{26}$ 


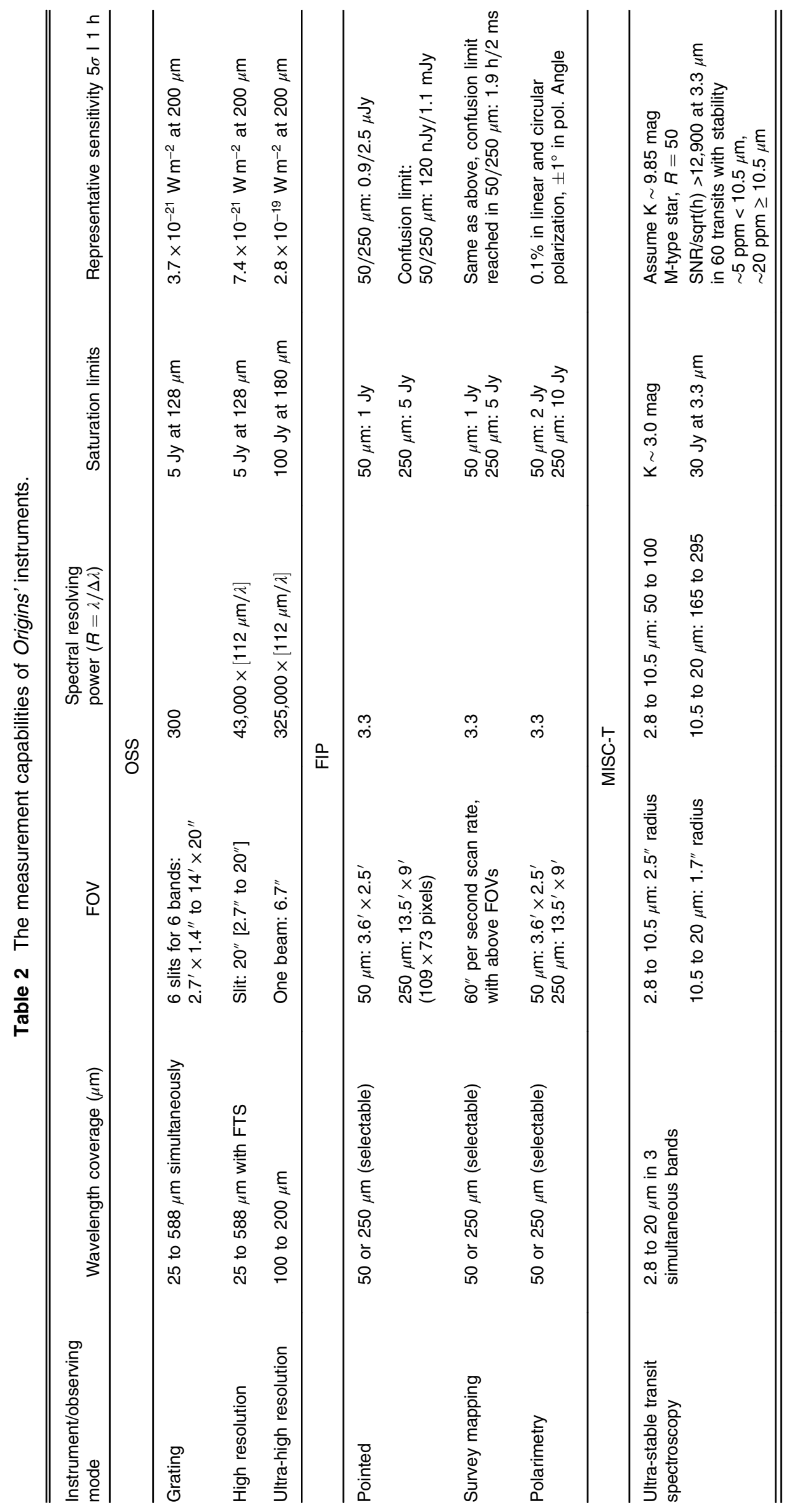




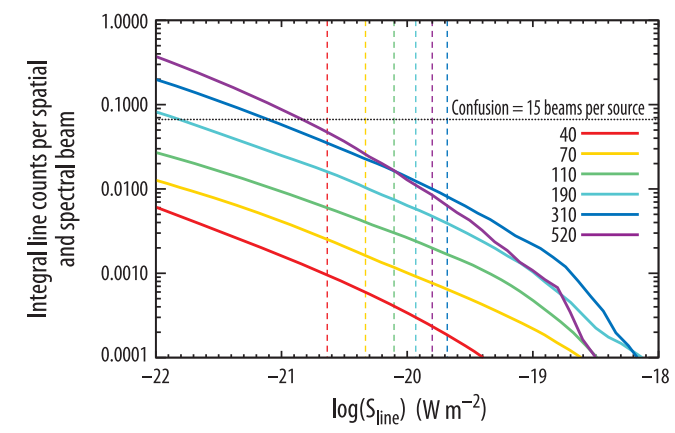

Fig. 7 Integral spectral line counts per spatial beam and spectral resolution element (at $R=300$ ) for each Origins/OSS band (wavelengths in $\mu \mathrm{m}$; see legend). The detection limit of the deep survey in each band is shown by the vertical dashed lines. The nominal confusion limit for imaging, 15 beams per source, is shown as the dotted horizontal line. In all bands, the counts for the deep survey are well below the confusion limit.

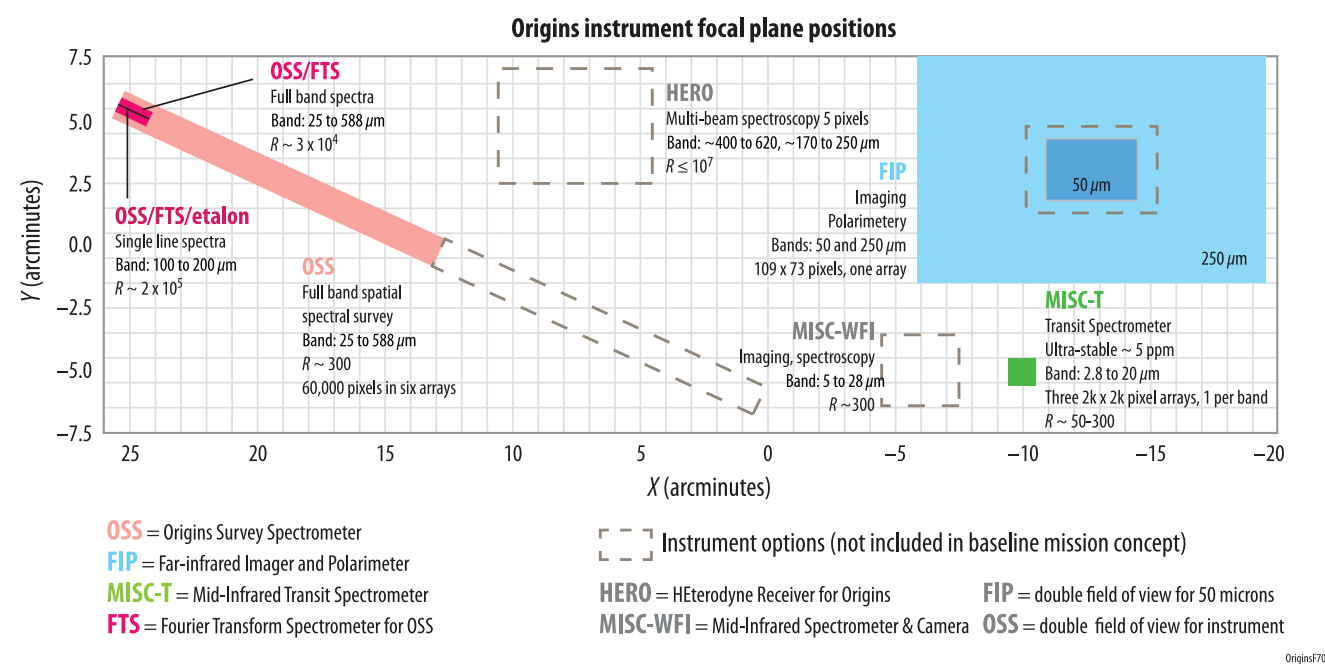

Fig. 8 The rms wavefront error over the Origins focal plane meets or exceeds performance requirements for each of the instruments. Items shown in gray (dashed lines) are enhancement options discussed in Sec. 3.4.1 and are not included in the baseline mission concept.

Space is allocated to OSS, FIP, and MISC-T in the telescope's focal plane, as shown in Fig. $8 .{ }^{11}$ Figure 8 also shows how the focal plane can accommodate the enhancement options discussed in Sec. 3.4.1. Figure 9 compares the instruments in terms of the spectral resolving power they offer across the Origins spectral range.

To reach their required sensitivity levels, OSS and FIP incorporate next-generation detectors. Several promising detector technologies already exist, including transition-edge-sensor bolometers, kinetic inductance detectors, and quantum capacitance detectors, ${ }^{20-22}$ all of which operate at temperatures in the 30 to $50 \mathrm{mK}$ range. At the subassembly level, the lowest technology readiness level (TRL) is 3 for detectors of these types. Advanced detectors will enable Origins to make the first ever fast and wide-area photometric and spatial-spectral surveys in the far-IR.

In the mid-infrared, from 2.8 to $20 \mu \mathrm{m}$, Origins builds on the amazing discoveries anticipated from JWST. JWST will deliver extraordinary sensitivity, but transiting exoplanet spectroscopy was not a major design driver. The Origins STDT prioritized exoplanet biosignature detection in the atmospheres of Earth-like planets in the habitable zones of M dwarf stars in the important 


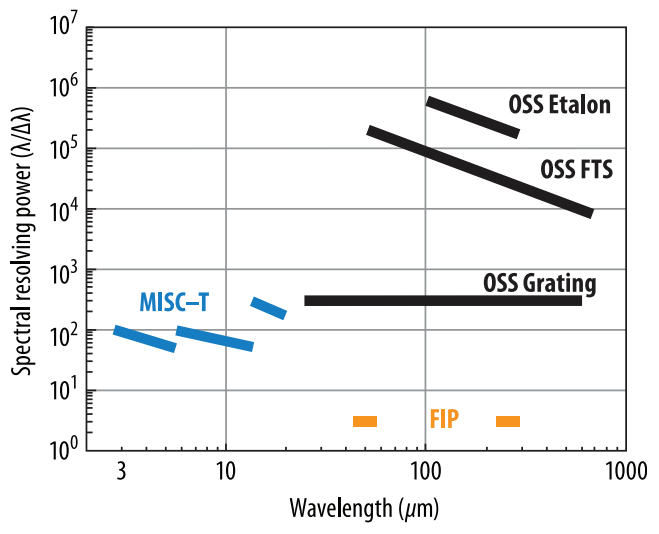

Fig. 9 MISC-T offers a spectral resolving power of order $10^{2}$ for transiting exoplanet spectroscopy and biosignature searching. In the far-IR, FIP provides broadband imaging in two wavelength bands, while OSS offers three modes of operation, with spectral resolving power ranging from 300 to $\sim 10^{6}$.

2.8 to $10 \mu \mathrm{m}$ range, and accordingly we established $5 \mathrm{ppm}$ as the required system-level stability for MISC-T. The Origins Technology Development Plan ${ }^{34}$ calls for investment in ultra-stable mid-IR detectors (next-generation HgCdTe arrays, Si:As impurity band conduction arrays, and transition-edge superconductor bolometer arrays) ${ }^{19}$ and offers multiple parallel development paths to reduce risk.

\subsubsection{Potential enhancements and potential descopes to the baseline concept}

The Origins baseline design can accommodate (in terms of mass, power, and volume) a fourth instrument, and the study team developed plans for the Heterodyne Receiver for Origins (HERO). ${ }^{16}$ If added to the mission, HERO would provide nine-beam measurements of any spectral line in the 111 to $617 \mu \mathrm{m}$ range, since it is continuously tunable. HERO would offer high spectral resolving power (up to $\sim 10^{7}$ ) and significantly enhance water-line observations of protoplanetary disks. With some modifications, HERO could vastly extend Event Horizon Telescope observations of supermassive black holes. ${ }^{35}$

Further instrument enhancement options exist in addition to HERO. We studied a Camera mode for MISC, the MISC wide-field imager (WFI); increased pixel counts and expanded footprints in the Origins telescope focal plane (Fig. 8) for OSS and FIP; and additional FIP bands at 100 and $500 \mu \mathrm{m}$. MISC-WFI would enable mid-IR imaging and spectroscopy $(R \sim 300)$ in the 5 to $28 \mu \mathrm{m}$ wavelength range.

While scientifically interesting, the STDT chose not to include the enhanced capabilities discussed in this section in the baseline design, to save costs. The STDT also noted, but chose not to accept, potential descopes relative to the baseline design concept. Descope options include eliminating instrument modes and decreasing the aperture diameter, which would erode science margin or degrade the observatory's science capability, as shown in Fig. 5.

\subsection{Saving Time During Integration and Test}

The Origins integration and test program ${ }^{17}$ is shorter than JWST's Phase D, in part because Origins has very few deployable elements. Additionally, Origins has a Cryogenic Payload Module (CPM) comprising a cold shield, telescope, and instrument package, within which each assembly is isothermal. The telescope, baffle, and instrument assembly are all cooled to $4.5 \mathrm{~K}$. The size of the fully integrated CPM allows cryogenic testing in Chamber A at NASA's Johnson Space Center, in accordance with NASA's preferred test-as-you-fly approach. By choosing thermally conductive, anhygroscopic materials for the isothermal components, the structure cools 


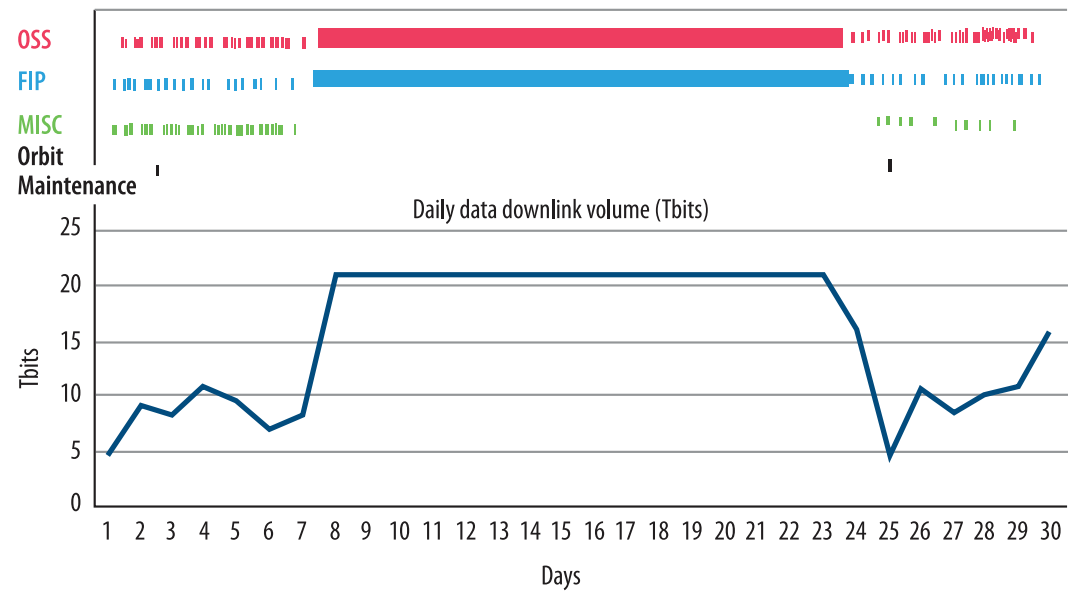

Fig. 10 A month in the life of Origins might include many observations with the three instruments OSS, FIP, and MISC-T and a wide survey with OSS and FIP, punctuated rarely by orbitmaintenance procedures. The daily data volume varies based on the types of observations scheduled. The upper part of the figure shows schematically when each of the instruments is in use during this notional month.

and warms rapidly during thermal vacuum testing. This saves schedule time and decreases the danger of water adsorbing onto sensitive surfaces.

\subsection{Additional Design Features}

Origins operates in a quasi-halo orbit around the Sun-Earth L2 point and transmits 21 Tbits per day of science data to the ground via optical communication at 1 Gbps. Optical communication is currently the state of the art, but undergoing rapid development and deployment. This technology will be mature and very well established before the start of Origins in 2025. Command and telemetry support is provided by heritage S-band transponders.

The minimum mission lifetime is 5 years, and the design lifetime is 10 years. The observatory design allows robotic servicing, which could enable future instrument upgrades and propellant replenishment to extend the mission beyond 10 years.

Figure 10 shows 30 days in the life of the Origins Space Telescope, during which the observatory executes the longest planned observation, a 16-day mapping observation by FIP and OSS. On average, Origins will spend $89 \%$ of the time collecting science data. The remaining time will be spent on slewing to new targets and settling before observations begin (6\%), instrument calibration (2\%), and smaller fractions of time on data transfer, station-keeping, momentum management, and in safe mode.

\subsection{Schedule}

Figure 11 shows a condensed version of the Origins mission development schedule from Phase A into Phase E. Scheduled milestones and key decision points are consistent with NASA Procedural Requirements (NPR-7120.5) and formulation and development for Class A missions. The duration of each of Phases A through D is comparable to the corresponding formulation and development times of previous large missions of similar complexity. The schedule supports an April 2035 launch following 10 years of development. The project plan provides 12.7 months of funded schedule reserve along the critical path, exceeding by 1.9 months the required reserve according to Goddard Procedural Requirements 7120.7B "Funded Schedule Margin and Budget Margin for Flight Projects" for Phases C and D (total duration 6.25 years). The schedule allows time for transportation to and from special integration and test facilities. Much of the design and development work progresses through parallel efforts, and the critical path runs through the most complex instrument, OSS. The plan includes 5 years of mission operations after launch, and an option to extend Phase E to 10 years. 


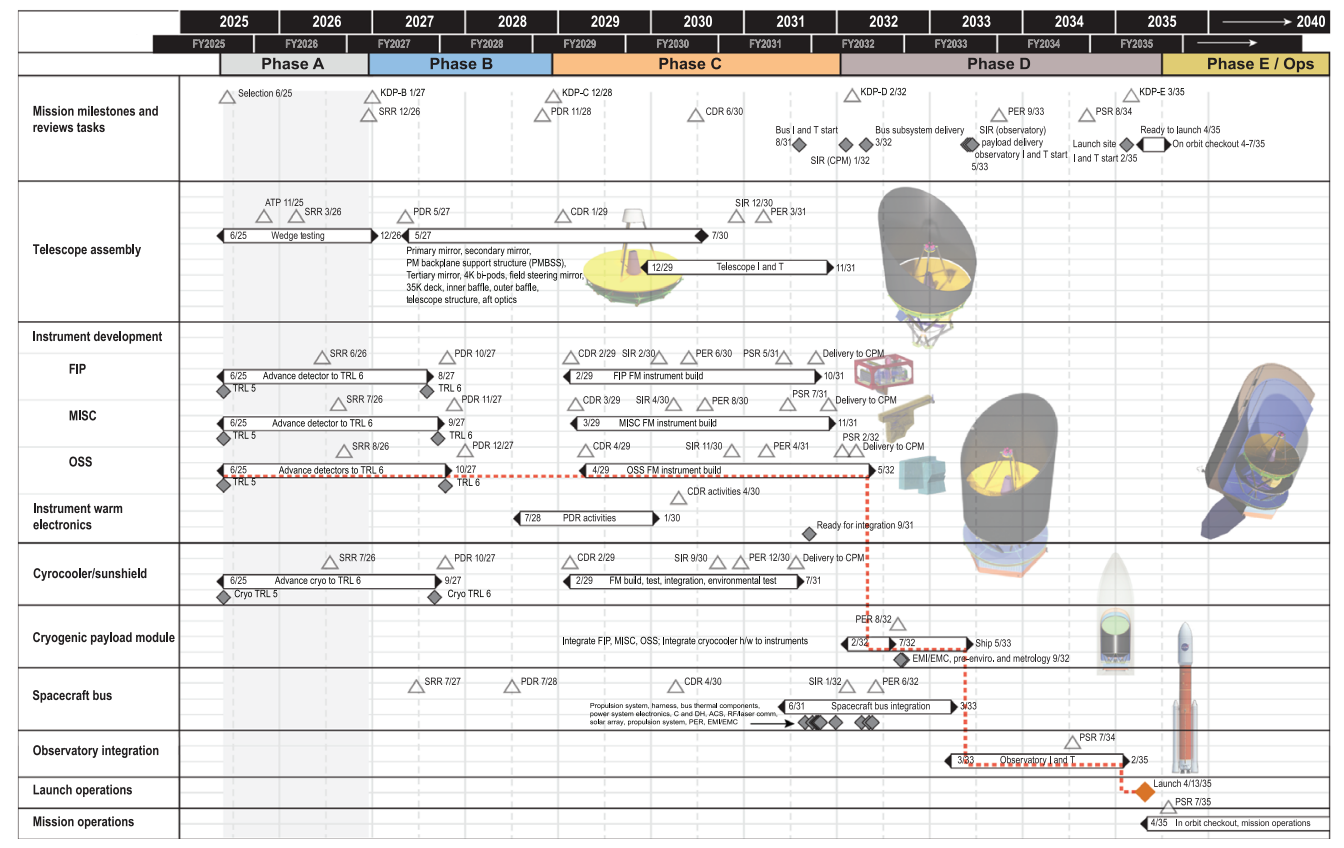

Fig. 11 Top-level schedule, showing key mission milestones, culminating in a 2035 launch.

\subsection{Estimated Cost}

The Origins lifecycle mission cost is estimated to be in the range US $\$ 6.7 \mathrm{~B}$ to $\$ 7.3 \mathrm{~B}$ at the $50 \%$ and $70 \%$ confidence levels, respectively. This estimated cost includes margins and reserves that meet NASA standards for a mission in preformulation, and covers mission Phases A through E, assuming no foreign contributions. The cost is given in Fiscal Year 2020 dollars. The cost estimate will evolve until the mission Preliminary Design Review (PDR). NASA Goddard Space Flight Center's (GSFC) Cost Estimating and Modeling Analysis (CEMA) office developed this cost estimate using the commercially available PRICE-H parametric cost modeling tool. The cost estimate is based on a detailed Master Equipment List (MEL) and a detailed Integrated Master Schedule (IMS), and it assumes that all components have matured to TRL 6 by mission PDR. The Origins Space Telescope Technology Development Plan begins in pre-Phase $\mathrm{A}$ and describes the maturation of all mission-enabling technologies (detectors ${ }^{19-23}$ and cryocoolers ${ }^{18}$ ) on this timeline and reports the cost of technology maturation. ${ }^{34}$ The estimated cost of technology development in pre-Phase A is $\$ 156 \mathrm{M}$ in real-year dollars. The mission cost estimate given above includes mission definition and development, the flight segment, the ground segment, and mission and science operations for 5 years. The launch cost $\$ 500 \mathrm{M}$ for the SLS launch vehicle, as advised by NASA Headquarters) is also included. NASA GSFC's Resource Analysis Office (RAO) independently estimated the mission cost using a top-down parametric approach. RAO and CEMA were firewalled from each other, but they both referred to the same MEL and IMS. The RAO and CEMA cost estimates agree to within the estimated uncertainty. The Origins mission design has not yet been fully optimized, and optimization is expected to lead to cost savings. Optimization is planned as a Phase A activity. Japan and several ESA member nations have significant relevant expertise and have demonstrated interest in the Origins mission through participation in the study. Foreign contributions are expected to reduce NASA's share of the mission cost.

\section{Estimated Performance}

With its next-generation detectors and cryocooled optical system, we estimate that Origins will be 1000 times more sensitive than prior far-IR missions, as shown in Fig. 12. Origins has only 2.8 times the collecting area of Herschel, but it is much colder: $4.5 \mathrm{~K}$ versus Herschel's $80 \mathrm{~K}$ telescope, which means Origins has a much lower thermal background and can deliver much 


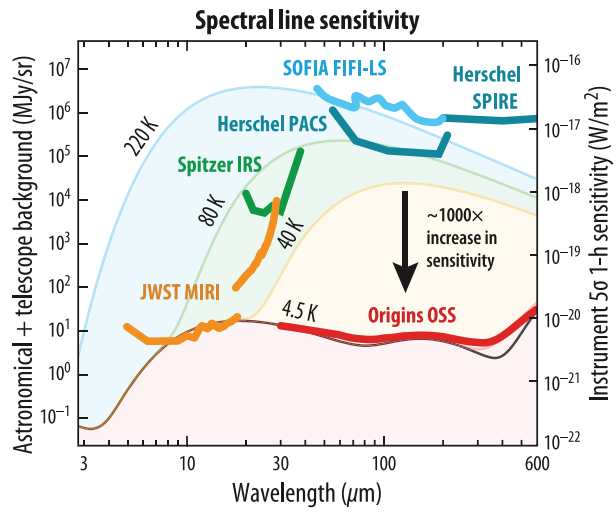

Fig. 12 Origins taps into a vast, unexplored scientific discovery space, defined by three-orders-ofmagnitude improvement in sensitivity relative to all previously flown far-IR observatories, and with superlative sensitivity bridging a wavelength gap between the JWST Mid-Infrared Instrument (MIRI) in orbit and ALMA on the ground. With next-generation detectors and a temperature of $4.5 \mathrm{~K}$, Origins' sensitivity is limited by astronomical background photon noise (lower black curve). SOFIA (220 K), Herschel (80 K), and JWST (40 K) are shown for comparison with Origins (4.5 K).

higher sensitivity. Similarly, the Earth's warm atmosphere limits the sensitivity of the Stratospheric Observatory for Infrared Astronomy (SOFIA), ${ }^{36}$ the only existing facility with significant wavelength overlap with Origins.

Origins was designed for agility, enabling wide-area surveys and solar-system object tracking, and deep targeted observations. The estimated time to survey a $1 \mathrm{deg}^{2}$ area photometrically to a depth of $1 \mathrm{mJy}(5 \sigma)$, or spectroscopically to a depth of $10^{-19} \mathrm{~W} \mathrm{~m}^{-2}(5 \sigma)$, is shown in Figs. 13(a) and 13(b), respectively. Figure 14 shows the importance of the far-IR spectral range for discerning the physical conditions in galaxies over a wide range of redshifts and their corresponding cosmic look-back times. Surveys conducted with FIP and OSS will enable the characterization of millions of galaxies of different types and evolutionary states.

The higher spectral resolving power modes of OSS are designed to enable measurements of water and total gas mass in protoplanetary disks. Resolving power in the tens of thousands is needed to detect the $\mathrm{H}_{2} \mathrm{O}$ and $\mathrm{HD}$ spectral lines above a bright continuum, while order-ofmagnitude greater resolution is required to measure line profiles and derive protoplanetary disk gas distributions using the line-tomography method. In the wavelength range 30 to $588 \mu \mathrm{m}$,

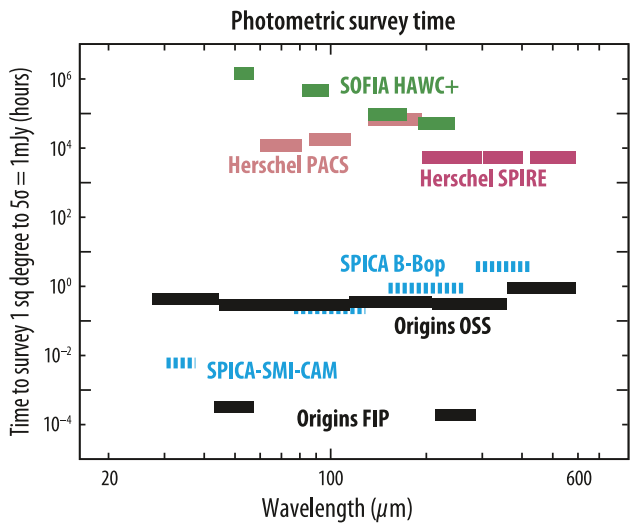

(a)

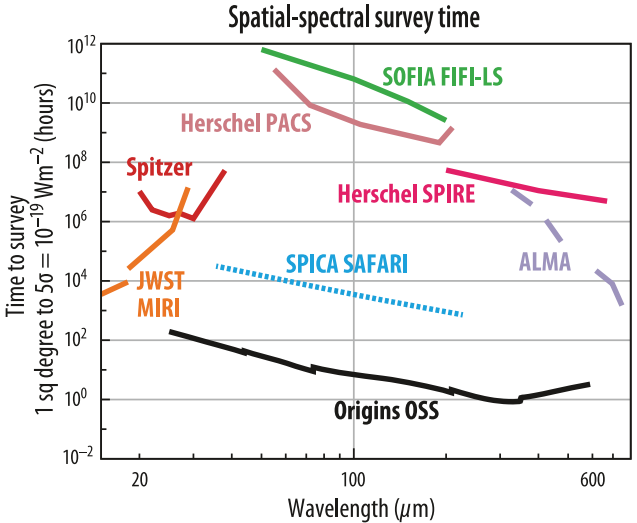

(b)

Fig. 13 With its great improvement in far-IR sensitivity, Origins will be able to map wide areas with instruments that provide (a) imaging (FIP) or (b) moderate spectral resolving power (OSS), enabling heretofore impossible surveys. The Space Infrared Telescope for Cosmology and Astrophysics (SPICA) is a less ambitious proposed and recently declined ESA M-class mission with similar science goals. Here, SPICA is assumed to have a 2.5-m telescope. 


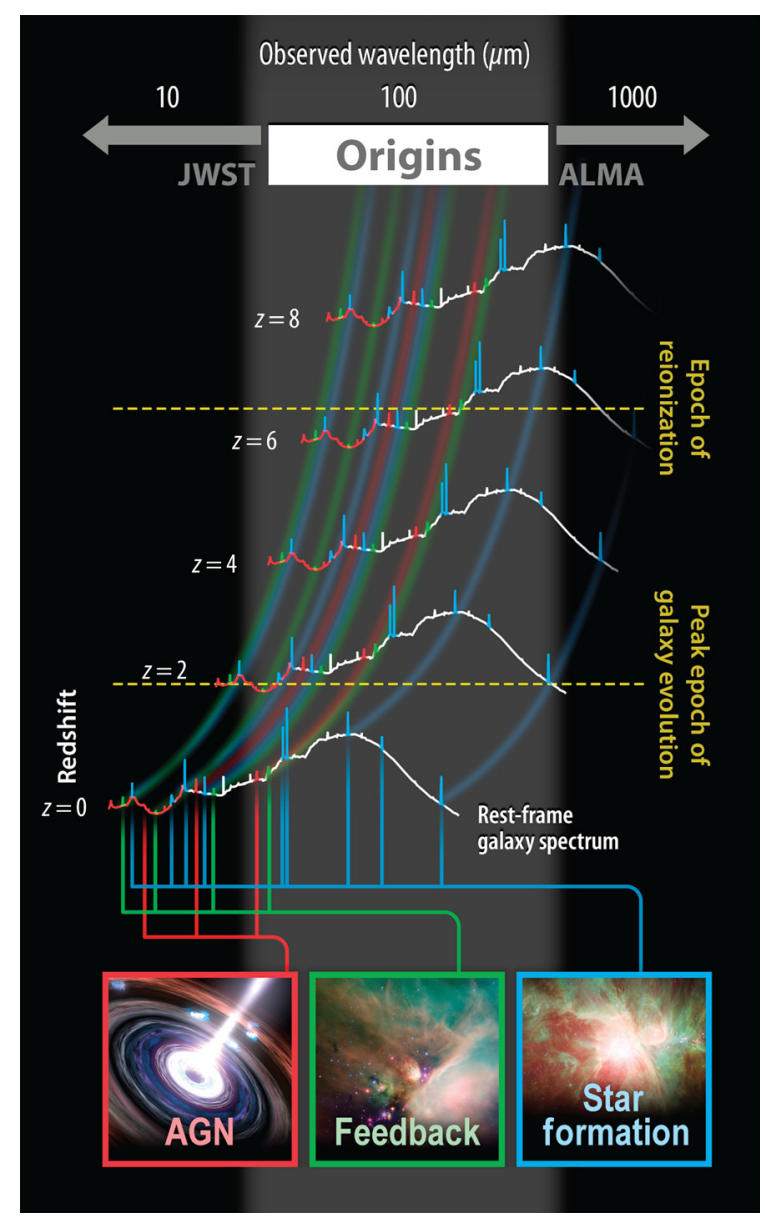

Fig. 14 Key spectral diagnostic features of active galactic nuclei (red), star formation (blue), and energetic feedback (green) move through the wide bandpass of the OSS with redshift $z$, or look-back time. Origins can measure these important processes over the entire history of galaxy evolution, filling in a key gap in wavelength and discovery space between JWST and ALMA. The spectrum of the nearby active galaxy Circinus is used as an example template here.

the Origins angular resolution will be $1.3(\lambda / 30 \mu \mathrm{m})$ arc sec, which is not adequate to resolve the disks spatially.

The Origins MISC-T instrument will be sensitive to $\mathrm{CO}_{2}$ and the biosignature pairs $\left(\mathrm{O}_{3}+\mathrm{CH}_{4}\right)$ and $\left(\mathrm{O}_{3}+\mathrm{N}_{2} \mathrm{O}\right)$ in the atmospheres of transiting exoplanets around late-type stars (Fig. 15). (Origins Guest Observers may wish to propose observations of earlier spectral-type stars, but we expect the potentially habitable planets they host will be exceedingly difficult to detect due to their greater distances from the host stars, and correspondingly longer elapsed time between transits.) JWST will make pathfinding observations, but assuming noise floors of 20 and $30 \mathrm{ppm}$ for the instruments/modes NIRSpec/G395H and MIRI/Low-Resolution Spectroscopy, respectively, JWST will only be sensitive to $\mathrm{CO}_{2}$ at $3.6 \sigma$ or more. The assumed JWST noise floors are current best estimates based on ground testing; the actual detector performance will not be known until JWST operates in space, and it could be better than assumed.

The Origins baseline design, characterized by the parameters shown in Table 1, carries significant margin between science-driven measurement requirements and estimated performance to assure a successful science mission.

\section{Summary}

The Origins Space Telescope was designed to answer three important science questions: 

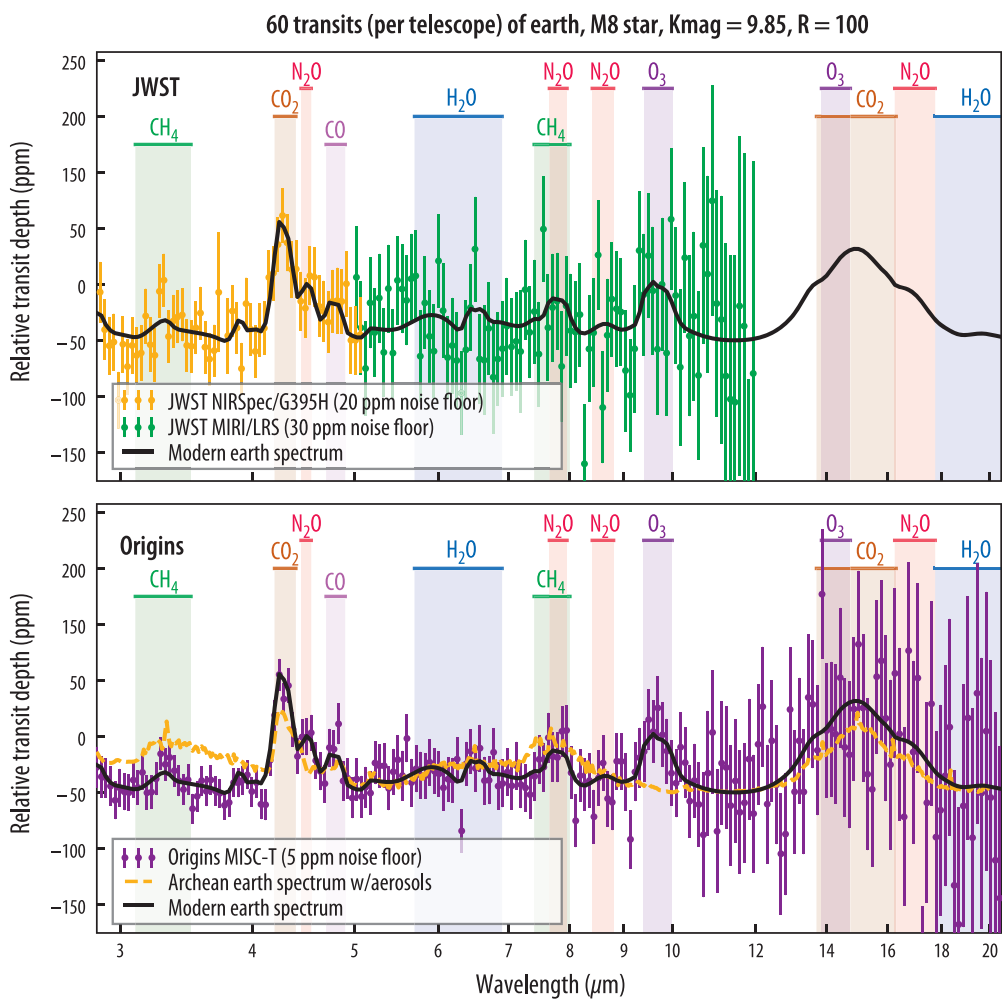

Fig. 15 With its broad wavelength coverage and anticipated noise floor of 5 ppm, MISC-T is designed to detect key habitability indicators and biosignature gases. Simulated transmission spectra are shown for a Transiting Planets and PlanetesImals Small Telescope (TRAPPIST)1e-like planet, with an Earth-like composition (60 transits, $R=100)$, comparing JWST (top) and Origins (bottom) measurements, based on current best estimates for their respective instrument noise floors (20 to 30 ppm for JWST and 5 ppm for Origins).

- How do galaxies form stars, make heavy elements, and grow their central supermassive black holes from reionization to today?

- How do the conditions for habitability develop during the process of planet formation?

- Do planets orbiting M dwarf stars support life?

The Origins STDT, in consultation with many members of the astronomical community, developed a detailed Science Traceability Matrix that flows from these questions to three scientific objectives per question, and then to instrument and telescope measurement requirements. ${ }^{9}$

The objectives are achievable with a large (5.9-m diameter) single-aperture telescope cryocooled to $4.5 \mathrm{~K}$, next-generation detector arrays, and three science instruments operating in the wavelength range 2.8 to $588 \mu \mathrm{m}$ and offering spectral resolving power from 3 to $3 \times 10^{5}$. Taking advantage of new launch-vehicle capabilities, the Origins study team developed a lowrisk (with very few deployments) mission concept that borrows its thermal architecture from the highly successful Spitzer Space Telescope, but uses mechanical cryocoolers instead of expendable cryogen. Origins is designed to operate for a minimum of 5 years in a quasi-halo orbit around the Sun-Earth L2 point, with a 10-year mission lifetime goal and an option for robotic servicing to replace instruments and extend the mission beyond 10 years.

To achieve its scientific objectives, Origins will be three orders of magnitude more sensitive than any previously flown far-IR telescope, agile enough to enable wide-area imaging and spectroscopic surveys, and its mid-IR instrument will be stable enough to measure biosignatures in transiting exoplanets. Leaps in measurement capabilities of this magnitude are very rare in astronomy and have always led to new discoveries and answers to questions that had not even been imagined when the telescopes or facilities were conceived. Thus, we expect Origins to enable astronomers in the 2030s to answer the compelling questions that motivate the mission and also to ask and answer new questions not yet imagined. 


\section{Acknowledgments}

We took excerpts from the SPIE Proceedings paper "The Origins Space Telescope," in Proc. SPIE, 11115-25 (2019) and from the Origins Space Telescope Mission Concept Study Final Report. The authors are grateful to the many institutions that sponsored and contributed to the successful Origins Space Telescope mission concept study. To enable the community to prepare for the 2020 Decadal Survey, the National Aeronautics and Space Agency, NASA, sponsored studies of four large mission concepts, of which Origins was one. We thank NASA for funding these studies. NASA's Goddard Space Flight Center (GSFC) contributed substantial additional labor support, which enabled us to explore options, make well-informed engineering decisions, and develop an executable mission concept. The Japan Aerospace Exploration Agency, JAXA, the Canadian Space Agency, CSA, and a Centre National d' Études Spatiales (CNES)-led European consortium actively participated in the study, with each enabling their team members' travel to study team meetings and concurrent engineering sessions. JAXA and the European consortium each contributed an instrument design. Domestic study participants included many academic institutions, several NASA centers (Ames Research Center and Marshall Space Flight Center, as well as GSFC), the Caltech Jet Propulsion Laboratory, and industry (Ball Aerospace, Northrop-Grumman, Lockheed-Martin, and L3 Harris), as reflected in the authors' affiliations. Finally, the authors wish to express their deep gratitude to the hundreds of community members worldwide who contributed to the Origins mission concept study by sharing their thoughts on science priorities, reviewing the science case and engineering designs, developing graphics, formatting reports, taking notes, managing study resources, and making travel arrangements. It took a village. Finally, we thank three JATIS reviewers, whose feedback on the manuscript led to significant improvements.

\section{References}

1. G. L. Pilbratt et al, "Herschel space observatory. An ESA facility for far-infrared and submillimetre astronomy," Astron. Astrophys. 518, L1-L6 (2010).

2. G. Neugebauer et al., "The Infrared Astronomical Satellite (IRAS) mission," Astrophys. J. Lett. 278, L1-L6 (1984).

3. N. W. Boggess et al., "The COBE mission-its design and performance two years after launch," Astrophys. J. 397, 420 (1992).

4. M. F. Kessler et al., "The Infrared Space Observatory (ISO) mission," Astron. Astrophys. 315, L27-L31 (1996).

5. M. W. Werner et al., "The Spitzer space telescope mission," Astrophys. J. Suppl. 154, 1-9 (2004).

6. H. Murakami et al., "The infrared astronomical mission AKARI," Publ. Astron. Soc. Jpn. 59, S369 (2007).

7. J. Gardner et al., "The James Webb space telescope," Sp. Sci. Rev. 123, 485 (2006).

8. D. Leisawitz et al., "The Origins Space Telescope: trades and decisions leading to the baseline mission concept and future study topics," J. Astron. Telesc. Instrum. Syst., 7(1) (2021).

9. M. Meixner et al., "Origins Space Telescope: science to design traceability," J. Astron. Telesc. Instrum. Syst., 7(1) (2021).

10. M. DiPirro et al., "The cryo-thermal architecture of Origins," J. Astron. Telesc. Instrum. Syst., (2020).

11. J. Corsetti et al., "Optical design of the Origins Space Telescope," J. Astron. Telesc. Instrum. Syst., (2020).

12. C. Sandin et al., "Materials evaluation for the Origins Space Telescope," J. Astron. Telesc. Instrum. Syst., 7(1) (2021).

13. C. M. Bradford et al., "The Origins Survey Spectrometer (OSS): revealing the hearts of distant galaxies and forming planetary systems with ultrasensitive far-IR spectroscopy," J. Astron. Telesc. Instrum. Syst., 7(1) (2021).

14. J. Staguhn et al., "The Far-infrared Imager and Polarimeter (FIP) for the Origins Space Telescope,” J. Astron. Telesc. Instrum. Syst., 7(1) (2021). 
15. I. Sakon et al., "The Origins Mid-Infrared Spectrometer Camera (MISC) baseline and upscope," J. Astron. Telesc. Instrum. Syst., 7(1) (2021).

16. M. Wiedner et al., "HEterodyne Receiver for Origins (HERO)," J. Astron. Telesc. Instrum. Syst., 7(1) (2021).

17. S. Petro et al., "Origins Space Telescope Integration and Testing," J. Astron. Telesc. Instrum. Syst. 6(4), 041502 (2020).

18. M. DiPirro et al., "Cryocooling technologies for the Origins Space Telescope," J. Astron. Telesc. Instrum. Syst., 7(1) (2021).

19. T. Roellig et al., "Mid-infrared detector development for the Origins Space Telescope," J. Astron. Telesc. Instrum. Syst., 6(4) (2020).

20. P. Echternach et al., "Large array of low frequency readout quantum capacitance detectors," J. Astron. Telesc. Instrum. Syst., 7(1) (2021).

21. S. Hailey-Dunsheath et al., "MKID detectors," J. Astron. Telesc. Instrum. Syst., 7(1) (2021).

22. J. E. Sadleir et al., "The state-of-the-art for TESs in the Origins band," J. Astron. Telesc. Instrum. Syst., 7(1) (2021).

23. E. Wollman et al., "Recent advances in superconducting nanowire single-photon detector technology for exoplanet transit spectroscopy in the mid-infrared," J. Astron. Telesc. Instrum. Syst., 7(1) (2021).

24. D. C. Bradley et al., "Advancements of digital signal processing hardware and algorithms enabling the Origins Space Telescope," J. Astron. Telesc. Instrum. Syst., 7(1) (2021).

25. P. A. Lightsey et al., "Stray light overview for the Origins Space Telescope," Proc. SPIE 10698, 1069845 (2018).

26. H. Matsuo et al., "A new concept for spectrophotometry of exoplanets with space-borne telescopes," Astrophys. J. 823, 139 (2016).

27. "Atacama Large Millimeter/submillimeter Array," https://www.almaobservatory.org/en/ home/ (accessed 11 December 2020).

28. "Vera C. Rubin Observatory," https://www.lsst.org (accessed 11 December 2020).

29. "Nancy Grace Roman Space Telescope," https://roman.gsfc.nasa.gov (accessed 11 December 2020).

30. T. H. Zurbuchen, "Explore science 2020-2024: a vision for science excellence," NASA's 2020 Strategic Plan for Science, https://science.nasa.gov/about-us/science-strategy.

31. M. G. Hauser et al., "The COBE diffuse infrared background experiment search for the cosmic infrared background. I. Limits and detections," Astrophys. J. 508, 25 (1998).

32. M. Betherman et al., "The impact of clustering and angular resolution on far-infrared and millimeter continuum observations," Astron. Astrophys. 607, 89 (2017).

33. M. Bonato et al., "Origins space telescope: predictions for far-IR spectroscopic surveys," Publ. Astron. Soc. Aust. 36, e017 (2019).

34. "Origins Space Telescope: Documents," https://asd.gsfc.nasa.gov/firs/docs/ (accessed 11 December 2020).

35. D. W. Pesce et al., "Extremely long baseline interferometry with Origins Space Telescope," Astro2020 Decadal Survey APC white paper, arxiv: 1909.01408 (2019).

36. "SOFIA," https://www.nasa.gov/mission_pages/SOFIA/overview/index.html (accessed 11 December 2020).

Edward Amatucci has developed everything from robotic devices to scientific instruments for space missions over the past 38 years as an engineer, and currently is an instrument systems engineer as a contractor for NASA Goddard Space Flight Center. He earned his bachelor of science degree in mechanical engineering from the University of Maryland, a master of engineering in mechanical engineering, and master of science in technical management from Johns Hopkins University.

Jonathan Arenberg is the chief engineer for Space Science Missions at Northrop Grumman Space Systems. He has worked on the Chandra X-ray Observatory, the Starshade, James Webb Space Telescope, technology development, and numerous mission studies, including Origins. His work experience also includes high energy lasers and other optical technologies. $\mathrm{He}$ is the author of over 200 conference presentations, papers, a book and book chapters, holds 13 patents and is an SPIE Fellow. 
Cara Battersby is an assistant professor of physics at the University of Connecticut. She received her $\mathrm{PhD}$ from the University of Colorado at Boulder, then held Submillimeter Array and National Science Foundation Postdoctoral Fellowships at the Harvard Smithsonian Center for Astrophysics. She is specialized in star formation in our galaxy'scenter. Her research group combines observational surveys with numerical simulations to understand the complex interplay of physics involved in star formation in this extreme environment.

Raymond Bell is a consultant. He is a retired senior fellow and chief scientist of the Lockheed Martin Advanced Technology Center. He began a long career in astrophysics living and working at the Cosmic Ray Observatory on Mt. Lemmon in southern Arizona, and has participated in many space- and ground-based missions for NASA and OGAs.

Charles M. Bradford obtained his bachelor's in physics at Stanford University in 1994 and his doctorate in astronomy and instrumentation at Cornell in 2001. He held a Millikan postdoctoral fellowship at Caltech from 2001-2003 and has been on the science staff at the Jet Propulsion Laboratory since that time. He has led the development and fielding of new submillimeter and millimeter-wave instrumentation on mountain-top site, using the datasets to study interstellar medium conditions in galaxies near and far. Current projects include a balloon-borne far-IR spectrometer, and on-chip mm-wave spectrometer, a mm-wave line intensity mapping instrument. He is also invested in developing concepts and the necessary detector systems for cryogenic space far-IR astrophysics missions.

Damon Bradley is head of Instrument Electronics Development Branch and founder of the Digital Signal Processing Technology Group at NASA Goddard Space Flight Center, where he supervises the research and development work of more than 70 engineers and 30 projects. $\mathrm{He}$ is also co-investigator of three different spaceflight spectrometer systems and serves as an adjunct professor in the Electrical and Computer Engineering Department at the University of Maryland, Baltimore County.

Denis Burgarella is an astronomer in the Laboratoire d'Astrophysique de Marseille, AixMarseille Universityn France. His preferred topic is the formation and evolution of galaxies and the quest to detect and understand the first stars and the first dust grains in the universe, from multi-wavelength data. He is at the origin of the SED modeling and fitting code CIGALE. $\mathrm{He}$ also is the present president of the IAU Division Cosmology and Galaxies.

Ruth Carter was a systems engineer and space flight mission development manager at the NASA Goddard Space Flight Center from 1986 to 2019 (retired, 2019). She managed and led a team to design and develop flight systems, including Hubble Space Telescope Servicing Missions, Tropical Rainfall Measuring Mission, and the Origins Space Telescope mission study. She received her BS and MS degrees in nuclear engineering from the University of Maryland at College Park.

Elvire De Beck is assistant professor at Chalmers University of Technology and is currently funded by the National Swedish Space Agency. She received her MSc and PhD degrees from KULeuven, Belgium. She was a fellow at the Max Planck Institute for Radio Astronomy in Bonn, Germany, after which she took positions as Atacama Pathfinder Experiment staff astronomer and postdoctoral researcher at Chalmers University of Technology. Her research relates mainly to evolved stars, their outflows, and mass-loss mechanisms.

Michael DiPirro received a $\mathrm{PhD}$ in low-temperature physics from the State University of New York at Buffalo, and a one-year National Research Council Postdoctoral Fellowship at the National Institute of Standards and Technology. Then, he joined NASA Goddard in 1980. He has worked on a number of Astrophysics missions over the last 40 years, including the Cosmic Background Explorer (COBE), Astro-E, -E2, and -H, XRISM, Spitzer, the Wide-field Infrared Explorer (WIRE), the Wide-field Infrared Survey Explorer (WISE), and JWST. Between COBE and Astro-E he was the principal investigator on the Superfluid Helium On-Orbit Transfer Flight Demonstration, and Co-I on a Cross Enterprise Technology Development Program to develop a new type of adiabatic demagnetization refrigerator. 
He is currently the technical lead and chief technologist for the Origins Space Telescope study for the 2020 Astrophysics Decadal Survey.

Matthew East innovates large optics as a lead opto-mechanical engineer at L3Harris Technologies in Rochester, New York. Since age one, he regularly attended telescope making conventions, learning about optical systems, fabrication, and metrology. He has patented inventions in additive manufactured optics and has published concepts for spaceborne astronomy missions. He holds a BSME from Rensselaer Polytechnic Institute and an MS in engineering management from Clarkson University. His goal is to drive the next generation of technology that enables breakthrough astronomical observations.

Kimberly Ennico has built infrared cameras, spectrometers, suborbital instruments, and lunar payloads. She has tested detectors at particle accelerators. She has served as SOFIA Project Scientist and New Horizons deputy project scientist. She has been a STDT member for Origins Space Telescope, authored more than 120 peer-reviewed papers, and delivered more than 50 invited technical talks and more than 70 public presentations. Asteroid 154587 Ennico is named for her. She is currently the Volatiles Investigating Polar Exploration Rover (VIPER) lunar rover deputy project scientist.

Louis Fantano earned his BS degree in chemical engineering from the University of Notre Dame in South Bend, Indiana, and joined Rockwell International as a thermal engineer on the Space Shuttle program in 1981. He began at NASA in 1991 and served as the lead thermal engineer for numerous flight programs, including the Landsat-8, Glory, Magnetospheric Multiscale Mission, Fermi Gamma-ray Space Telescope, Sub-millimeter Wave Astronomy Satellite, and Geostationary Operational Environmental Satellites flight projects.

Maryvonne Gerin is a senior scientist at the Centre National de la Recherche Scientifique (CNRS) France, has extensive experience in studies of the diffuse and dense interstellar medium, with special emphasis on the chemical properties of the matter using ground based or spaceborne radio telescopes. She led the Probing InterStellar Molecules with Absorption line Studies (PRISMAS) Herschel key program targeting hydrides in the diffuse interstellar medium. She is now the co-PI of the Institut de Radioastronomie Millimétrique (IRAM) Outstanding Radio-Imaging of Orion B (ORION-B) large program.

Thomas P. Greene received his PhD in astronomy from the University of Arizona in 1991 and held positions at the University of Hawaii Institute for Astronomy and Lockheed Martin before joining NASA in 1998. He is an astrophysicist at NASA's Ames Research Center in Mountain View, California. He is a co-investigator on the JWST NIRCam instrument and a member of the JWST Mid-Infrared Science Team and conducts observational studies of young stars and extrasolar planets.

George Harpole, UCLA PhD in engineering 1980, has worked for TRW, Inc. and Northrop Grumman for the 40 years since. George has 14 U.S. patents on a wide range of topics, chemical reactors for lasers, solid state lasers, cryocoolers, thermal storage, automotive steering noise reduction, etc. His specialty areas are heat transfer and fluid mechanics. He developed nongray radiative thermal modeling for JWST.

Joseph Howard received his BS degree in physics from the US Naval Academy in Annapolis, Maryland, and his PhD in optical design from The Institute of Optics, University of Rochester, in Rochester, New York. He now serves as an optical designer for NASA, working on projects including the James Webb Space Telescope, the Roman Space Telescope, Origins, and the other future space missions.

John S. Knight is a senior staff consultant in Missions Systems Engineering at Ball Aerospace. His current focus is payload architecture for large space systems. He has been involved with segmented space telescopes for over $15+$ years, concentrating on system engineering, modeling, test design for ground-based verification and flight commissioning. 
David Leisawitz is NASA study scientist for the Origins Space Telescope and is most interested scientifically in the development of habitable conditions during planet formation. He was PI on the Space Infrared Interferometric Telescope mission concept study and served as NASA Goddard study lead for the Submillimeter Probe of the Evolution of Cosmic Structure. He was mission scientist for the Wide-field Infrared Survey Explorer and deputy project scientist for the COBE.

Paul Lightsey has 50+ years' experience including work on HST instruments, Webb, and studies for future large space telescopes. He has co-authored a book on systems engineering of astronomical telescopes. He has a BS degree from Colorado State University, a PhD from Cornell University, and has received the Distinguished Public Service Medal from NASA. $\mathrm{He}$ is a SPIE Fellow and a senior member of OSA.

Eric Mamajek is deputy program chief scientist of the NASA Exoplanet Exploration Program, officed at the Jet Propulsion Laboratory. His research interest is the formation and evolution of exoplanetary systems and their host stars.

John C. Mather is senior project scientist for the James Webb Space Telescope. As a 28-yearold postdoc, he led the proposal for the COBE, and served as its project scientist, leading the COBE team to success and to the Nobel Prize in physics (2006). He leads the science study team for the Orbiting Configurable Artificial Star to enable high performance adaptive optics on ground-based telescopes.

Margaret Meixner is director of the SOFIA Science Mission Operations center. She received her bachelor's degrees in electrical engineering and math (1987), University of Maryland, College Park, and her PhD in astronomy (1993), University of California, Berkeley. Her research includes infrared instrumentation and dust evolution in galaxies. She is the member of the JWST/ MIRI Science Team. She is the community co-chair of NASA's STDT for the Origins Space Telescope mission concept. Formerly, she was the JWST Project Scientist and Distinguished Astronomer at STScI. She is a fellow of the American Association for the Advancement of Science (AAAS).

Gary Melnick is a senior astronomer at the Harvard-Smithsonian Center for Astrophysics specializing in infrared/submillimeter spectroscopy and astrochemistry. For more than 45 years, he has conducted airborne, balloon-borne, and space-based observations focused on the main coolants of interstellar clouds and understanding the formation, abundance, and distribution of interstellar water. He was PI of the SWAS Explorer mission, Co-I on the Herschel mission, and science lead for the Ices Investigation on the upcoming Spectro-Photometer for the History of the Universe, Epoch of Reionization and Ices Explorer (SPHEREx) mission.

Stefanie Milam is an astrochemist with expertise in observations and spectroscopy at millimeter and submillimeter wavelengths and coordinating ground-based campaigns of cometary apparitions at multiple wavelengths. She also represents the planetary science community on a number of astrophysics missions and concepts including JWST, Roman Space Telescope, and the Origins STDT.

Jeffrey Olson received his physics PhD in 1993 from Cornell University, and has worked on mechanical cryocoolers at Lockheed Martin Space since joining the company in 1997. He is specialized in designing, building, and testing innovative, highly efficient, long life mechanical cryocoolers for space applications. He has served on the board of the International Cryocooler Conference, and holds seven patents and patents-pending in the field of cryocoolers.

Susanna Petro obtained her doctor's degree in physics from the University of Rome, Italy La Sapienza and has more than 30 years of experience in spacecraft and instrument systems design, test, and launch. She is the NASA/GSFC Flight Systems Integration and Test Staff Engineer. She previously worked at the NASA Johnson Space Center and at Thales Alenia Space in Europe in the development of satellites and payloads for telecommunications, science and exploration, and remote sensing. 
Alexandra Pope received her PhD from the University of British Columbia. She is an associate professor of astronomy at the University of Massachusetts, Amherst. She is an observational astronomer who specializes in infrared, (sub)millimeter, and radio observations of dusty galaxies at high redshift.

Thomas Roellig has worked in the NASA civil service as an astrophysicist at the NASA Ames Research Center since 1980 and is currently the chief of the astrophysics branch at Ames. His scientific research interests have spanned a wide range of infrared astronomy and astronomical instrumentation development. He has conducted research and published papers in infrared instrument development, solar science, solar system science, star formation, interstellar medium, and brown dwarf astronomy.

Douglas Scott received his BSc degree from the University of Edinburgh and $\mathrm{PhD}$ from the University of Cambridge. He is a professor of physics and astronomy at the University of British Columbia. He has co-authored more than 600 papers on a wide range of topics in cosmology, specializing in studies of the cosmic microwave background and submillimeter galaxies in the high-redshift universe.

Len Seals received his BS degree in physics from Grambling State University in Grambling, Louisiana, and his $\mathrm{PhD}$ in applied physics from the Georgia Institute of Technology, in Atlanta, Georgia. He now serves as an optical physicist and stray light analyst at NASA, where he is an associate branch head in the optics branch. He supports various projects, including the James Webb Space Telescope, the Roman Space Telescope, Lucy, Origins, and the other future space missions.

Kevin Stevenson is a staff astronomer at Johns Hopkins Applied Physics Laboratory and adjunct assistant professor in Johns Hopkins University's Department of Earth and Planetary Sciences. He is interested in characterizing the architectures and atmospheres of extrasolar planets to better understand their nature and origin; developing exoplanet mission concepts to measure the composition and chemical properties of nearby potentially habitable worlds; and building software packages and tools to enable exoplanet observations and atmospheric characterization.

Steven Tompkins is an engineer at NASA GSFC, specializing in the definition of operations concepts and ground system architectures for missions in the concept definition and formulation phase.

Martina C. Wiedner is a CNRS research scientist at Paris Observatory. She received her undergraduate degree from Karlsruhe University and her $\mathrm{PhD}$ from Cambridge University. She held a Submillimeter Array (SMA) postdoc at the Harvard-Smithsonian Center for Astrophysics and a post at the University of Cologne. She built and designed several heterodyne receivers, including the 1.4-THz CO N+ Deuterium Observation Receiver (CONDOR) receiver and 183-GHz water vapor monitors. She is interested in star formation and starburst galaxies.

Chi Wu is a mission system engineer at NASA/GSFC with experience ranging from communication satellites in private industry to NASA Science missions, from preparing proposals in response to announcements of opportunity to conducting mission studies, mission planning, and mission development from pre-Phase A to launch.

Biographies of the other authors are not available. 